Reprod. Nutr. Develop., 1988, 28 (3 B), 715-720

\title{
Régulation de l'expression des gènes $\alpha$ et $\beta$ de la tropomyosine au cours du développement du muscle pectoral chez le poulet
}

\author{
T. MEINNEL, D. LIBRI, Danièle GROS, M. Y. FISZMAN, Marguerite LEMONNIER
}

Unité de Biochimie, Département de Biologie moléculaire, Institut Pasteur, 25-28, rue du Dr Roux, 75724 Paris Cedex 15.

Summary. Regulation of the expression of $\alpha$ and $\beta$ tropomyosin genes in developing chicken pectoralis muscle.

Accumulation of mRNAs coding for $\alpha$ and $\beta$ skeletal tropomyosins was investigated using specific probes and normalized to muscle creatine kinase (M-CK) mRNA by slot-blot assays. In developing pectoralis muscle, the ratio of $\alpha \mathrm{TM}$ messenger/M-CK remained constant until hatching, at which time the messenger disappeared within a week. However, in the leg, this ratio remained constant until 8 days after hatching, whereafter it decreased progressively to reach $30 \%$ in the adult. The $\alpha \mathrm{TM} / \mathrm{M}-\mathrm{CK}$ ratios were almost the same in the embryonic leg and pectoralis muscle. After hatching, there was a large increase in pectoralis muscle $(\times 3$ at day $+4, \times 0$ at day +21$)$ whereas, the increase was less pronounced and more progressive in the leg ( $\times 3$ at day 21 ). Run-on assays showed that nuclei isolated from 15-day in ovo leg and pectoralis muscles had similar patterns of muscle specific gene transcription whereas post-hatched pectoralis muscle nuclei were shown to have a higher rate of $\alpha$ to $\beta$ tropomyosin gene transcription. These data are in accordance with the results obtained for protein analysis of leg and pectoralis muscles and support the notion that changes in the protein pattern of developing muscle can be relevant to coordinate regulation of gene transcription.

\section{Introduction.}

Les protéines contractiles identifiées dans la totalité de la masse du muscle pectoral embryonnaire sont les isoformes $\alpha$ et $\beta$ rapides de la tropomyosine (Roy et al., 1979 ; Matsuda et al., 1983) et une faible quantité de tropomyosine lente (Matsuda et al., 1983), les isoformes LC1 et LC2 rapides (les formes lentes sont très minoritaires) des chaînes légères de la myosine (Hoh, 1979 ; Obinata et al., 1980b ; Matsuda et al., 1983), une isoforme embryonnaire de la chaîne lourde de la myosine rapide (Bandman et al., 1982) et les deux classes de troponines $\mathrm{T}$ "Leg " et « Breast » (Obinata et al., 1980a; Matsuda et al., 1981).

Deux transitions interviennent pendant le développement : d'abord la ségrégation des isoformes lentes des protéines contractiles, dès le $16^{\mathrm{e}}$ jour in ovo, vers 
la partie antérieure du muscle pour former la zone rouge. Puis, après l'éclosion, intervient la disparition en une à deux semaines de l'isoforme $\beta$ de la tropomyosine et des troponines de type «Leg » (Matsuda et al., 1980a), I'apparition de LC3 (Hoh, 1979) ainsi que le remplacement de la forme embryonnaire de la chaîne lourde de la myosine par une forme néonatale (Bandman et al., 1982). De ces quatre événements qui sont synchrones, seul les trois premiers semblent coordonnés et étroitement associés à l'innervation : en effet, la dénervation du muscle pectoral de poussin nouveau-né n'interfère pas avec la séquence des transitions de la chaîne lourde de la myosine alors qu'elle entraîne le maintien d'un phénotype embryonnaire (Matsuda et al., 1984) pour les tropomyosines, les troponines $T$ et les chaînes légères rapides de la myosine. La possibilité d'analyser les mécanismes moléculaires mis en jeu par l'innervation, l'existence possible d'une coordination dans l'expression de plusieurs protéines contractiles au cours du développement font du muscle pectoral un modèle particulièrement intéressant. L'isoforme $\beta$ de la tropomyosine est un bon marqueur pour analyser ce système et le clonage des gènes $\alpha$ et $\beta$ (Libri et al., 1988; Fiszman et al., 1986) réalisé au laboratoire a apporté les sondes indispensables à l'analyse des différents niveaux de contrôle possibles: transcription du gène, maturation, stabilité et traductibilité des messagers.

\section{Matériel et méthodes.}

Les ARNs du muscle pectoral et des muscles de la patte ont été préparés à partir de tissus prélevés à différentes étapes du développement (Chirgwin et al., 1979). L'électrophorèse des ARN est effectuée dans un gel d'agarose $1 \%$ contenant de la formaldéhyde $1 \mathrm{M}$ (Maniatis et al., 1982). Après transfert sur nitrocellulose, les filtres sont hybridés avec des sondes radio-actives marquées par déplacement de coupure et exposés après rinçages. L'expression des messagers spécifiques des tropomyosines $\alpha$ et $\beta$ de muscle squelettique est quantifiée en slot-blot (Hardeman et al., 1986). Les résultats obtenus avec des sondes de radioactivités spécifiques comparables sont exprimés par rapport à la créatine kinase musculaire et ramenés à une même longueur de séquence.

Les noyaux sont préparés (Mellon et Bhorjee, 1982) après dilacération des tissus à $+4^{\circ}$, et purifiés (Schibler et al., 1983). Les expériences de run-on sont faites (Bentley et Groudine, 1986) avec des noyaux vérifiés en fluorescence (coloration au BET) et en microscopie électronique après le traitement à la RNase. Les ARN radiomarqués transcrits in vitro sont hybridés avec des plasmides recombinants correspondant à différentes protéines du programme myogénique.

\section{Résultats. Discussion.}

Les messagers accumulés au cours du développement du muscle pectoral et de la patte ont été analysés avec différentes sondes spécifiques des tropomyosines (extrémités 3 ' non-codantes des gènes (Libri et al., 1988 ; Fiszman et al., 
1986), ne révélant que les messagers $\alpha(1,4 \mathrm{~Kb})$ et $\beta(1,6 \mathrm{~Kb})$ squelettiques rapides) et avec des sondes correspondant à diverses protéines et enzymes du programme myogénique, dont la spécificité a été vérifiée en Northern.

L'expression des deux messagers $\alpha \mathrm{TM}$ et $\beta \mathrm{TM}$ au cours du développement a été évaluée par rapport à celle d'une sonde cADN (Rosenberg et al., 1985) de l'isoforme musculaire de la créatine kinase (M-CK) seule présente dans le muscle après différenciation (Lough et Bischoff, 1977). Dans le muscle pectoral, le messager $\beta$ est accumulé en plateau (fig. $1 \mathrm{a}$ ) et chute dès l'éclosion (noter la différence entre les points 20 jours in ovo et 1 jour après l'éclosion) ; dans la patte, un plateau est visible, jusqu'au $8^{\mathrm{e}}$ jour après l'éclosion, puis le signal du messager $\beta$ diminue ( $30 \%$ de la valeur maximum chez l'adulte). Pendant la période in ovo, le messager $\alpha$ est exprimé à des niveaux comparables dans la patte et le pectoral (fig. $1 \mathrm{~b}$ ), puis accumulé de façon différentielle après la naissance (à 21 jours $\times 3$ dans la patte, $\times 8$ dans le pectoral).

L'arrêt de l'accumulation du messager codant pour l'isoforme $\beta$ de la tropomyosine au cours du développement du mucle pectoral est donc à l'origine de la disparition de cette protéine. Le décalage observé entre les deux phénomènesest dû à la durée de vie à la fois du messager et de la protéine. L'accumulation concommitante du messager $\alpha$ pourrait être liée de façon coordonnée à la variation du messager $\beta$. En effet, dans le cas du pectoral, l'extinction totale du messager $\beta$ entraîne une augmentation très considérable du messager $\alpha$ alors que, dans la patte où persiste l'isoforme $\beta$ (Montarras et al., 1982), l'augmentation du messager $\alpha$ est modérée. Plusieurs mécanismes peuvent être impliqués dans cette régulation concertée: transcription des gènes, modification de l'épissage des messagers comme rapporté pour les isoformes LC1 et LC3 (Nabeshima et al., 1982), stabilité des messagers... Une éventuelle modification de maturation des messagers a pu être écartée grâce à l'utilisation, en Northern blots, de sondes $\beta$ ou $\alpha$, contenant des séquences communes aux messagers non musculaires et squelettiques, qui n'ont révélé que ces derniers.

La transcription des gènes $\beta$ et $\alpha \mathrm{TM}$ a alors été explorée grâce à l'analyse des ARN en cours de transcription dans des noyaux isolés (run-on). Les résultats obtenus à partir de noyaux de patte et de pectoral prélevés à 15 jours in ovo sont équivalents et des signaux sont observés pour toutes les sondes musculaires, indiquant la transcription de ces gènes; un signal est également observé pour l'isoforme $\beta$ de l'actine, témoignant une différenciation cependant encore incomplète. Les signaux obtenus à partir de noyaux de pectoral de poussin nouveau-né montrent une augmentation faible de la transcription du gène $\alpha \mathrm{TM}$ ainsi qu'une faible diminution de la transcription du gène $\beta T M$, visible tout le long du gène (il n'y a donc pas d'arrêt précoce de la transcription comme rapporté pour d'autres gènes (Bentley et Groudine, 1986).

L'ensemble de ces résultats suggère que les variations dans les quantités d'ARN accumulés soient directement liées à un contrôle transcriptionnel exercé, au cours du développement, par un ou plusieurs facteurs sur la transcription des gènes $\alpha$ ou $\beta$, en agissant au niveau des séquences régulatrices de chacun des gènes. Une autre hypothèse serait l'existence d'un contrôle en trans exercé, directement ou indirectement, par le produit d'un des gènes sur l'autre. Quoiqu'il

Reproduction, Nutrition, Développement, $n^{\circ} 3$ B. -4 

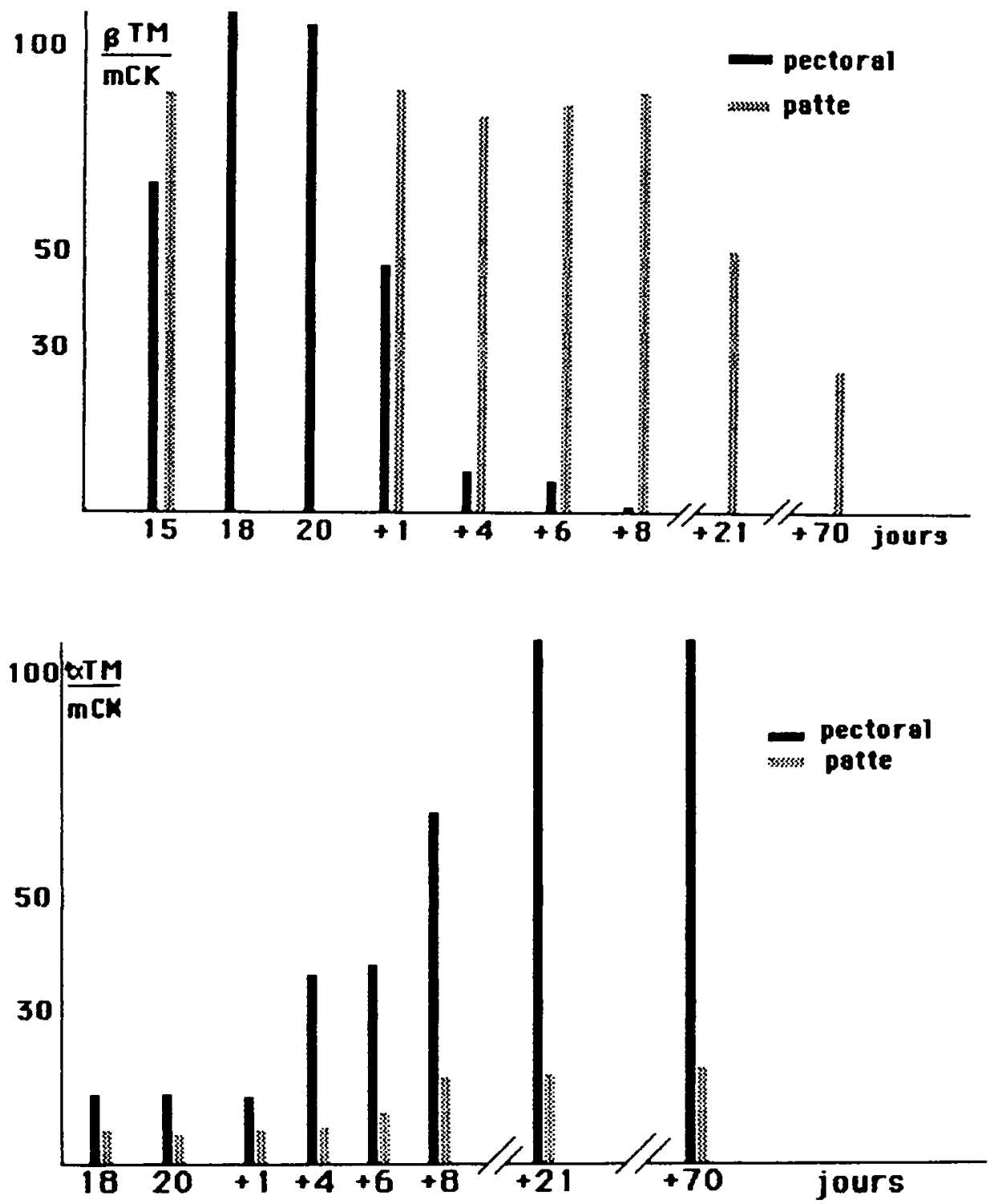

FIG. 1. - L'accumulation de messagers spécifiques des isoformes $\alpha$ et $\beta$ squelettiques de la tropomyosine de muscle strié rapide au cours du développement du muscle pectoralis major et des muscles de la patte, chez le poulet est déterminée avec des sondes spécifiques de radioactivités comparables, les résultats sont exprimés par rapport à la créatine kinase et ramenés à une même longueur de sonde : $1 \mathrm{a}$ : messager $\beta$-tropomyosine ; $1 \mathrm{~b}$ : messager $\alpha$-tropomyosine. 
en soit, il reste à définir les mécanismes qui entraînent une extinction totale du gène $\beta$ dans le pectoral, alors que ce même gène est coexprimé avec le gène $\alpha$ dans la patte, le PLD, etc...

La possibilité d'une expression concertée des tropomyosines et de certaines troponines $T$ serait également à explorer, mais la complexité des cADN codant pour les troponines $T$ tant chez le poulet (Cooper et Ordahl, 1984) que chez la caille (Hastings et al., 1985) ou le rat (Breitbart et al., 1985) n'a pas permis d'obtenir de sondes spécifiques des différentes isoformes. Quant aux chaînes légères de la myosine, elles sont produites par l'utilisation différentielle de deux promoteurs au cours du développement et si les variations de l'expression de ces deux isoformes coïncident dans le temps avec les modifications du phénotype des tropomyosines, il est vraisemblable que les modes de régulation de ces systèmes sont cependant différents.

$13^{e}$ Réunion du groupe Développement I.N.R.A., Cap d'Adge, 25-27 mai 1987.

Remerciements. - Ce travail a été financé par I'INSERM, le C.N.R.S., I'A.R.C., la Fondation pour la Recherche Médicale Française, la Ligue Française contre le Cancer, l'Association des Myopathes de France, la Muscular Dystrophy Association. Les auteurs remercient J. C. Perriard, B. Paterson, A. McLeod, C. Emerson pour le don de plasmides recombinants.

\section{References}

BANDMAN E., MATSUDA R., STROHMAN R.C., 1982. Developmental appearance of myosin heavy and light chain isoforms in vivo and in vitro in chicken skeletal muscle. Dev. Biol., 93. 508-518.

BENTLEY D.L., GROUDINE M., 1986. A block to elongation is largely responsible for decreased transcription of $\mathrm{c}-m y c$ in differentiated HL 60 cells. Nature, 321, 702-706.

BREITBART R. A., NGUYEN M. T., MEDFORD R. M., DESTREE A.T., MAHDAVI V., NADALGINARD B., 1985. Intricate combinatorial patterns of exon splicing generate multiple regulated troponin $\mathrm{T}$ isoforms from a single gene. Cell, 41, 67-82.

CHIRGWIN J. M., PRZYBYLA A. E., MacDONALD R., RUTTER W. J., 1979. Isolation of biologically active ribonucleic acid from sources enriched in ribonuclease. Biochemistry, 18 , 5294-5299.

COOPER T. A., ORDAHL C. P., 1984. A single troponin T gene regulated by different programs in cardiac and skeletal muscle development. Science, 226, 976-982.

FISZMAN M., KARDAMI E., LEMONNIER M., 1986. A single gene codes for gizzard and skeletal muscle $\alpha$-tropomyosin, 457-471. In C. EMERSON, D. A. FISHMAN, B. NADAL-GINARD, M. A. Q. SIDDIQUI, Conf. Proc. UCLA Molecular biology of development. Alan Liss Pub.

HARDEMAN E. C., CHIU C. P., MINTY A., BLAU H., 1986. The pattern of actin expression in human fibroblast $X$ mouse muscle heterokaryons suggests that human regulatory factors are produced. Cell, 47, 123-130.

HASTINGS K. E. M., BUCHER E. A., EMERSON C. P., 1985. Generation of troponin T isoforms by alternative RNA splicing in avian skeletal muscle. J. Biol. cell, 260, 13699-13703.

НОН J. F. Y., 1979. Developmental changes in chicken skeletal myosin isoenzymes. FEBS Lett., 98 , 267-270.

LIBRI D., LEMONNIER M., MEINNEL T., FISZMAN M., 1988. A single gene codes for the $\beta$-subunit of smooth and skeletal muscle tropomyosin in the chicken (à paraître).

LOUGH J., BISCHOFF R., 1977. Differentiation of creatine phosphokinase during myogenesis: quantitative fractionation of isozymes. Dev. Biol., 57, 330-344. 
MANIATIS T., FRITSCH E. F., SAMBROOK J., 1982. Molecular cloning. Cold Spring Harbor Lab. Pub., New York.

MATSUDA R., OBINATA T., SHIMADA Y., 1981. Types of troponin components during development of chicken muscle. Dev. Biol., 82, 11-19.

MATSUDA R., BANDMAN E., STROHMAN R. C., 1983. Regional differences in the expression of myosin light chains and tropomyosin subunits during development. Dev. Biol., 95, 484-491.

MATSUDA R., SPECTOR D., STROHMAN R. C., 1984. Denerveted skeletal muscle displays discoordinate regulation for the synthesis of several myofibrillar proteins. Proc. nat. Acad. Sci. USA, 81, 1122-1125.

MELLON I., BHORJEE J. S., 1982. Isolation and characterization of nuclei and purification of chromatin from differentiating cultures of rat skeletal muscle. Exp. cell Res., 137, 141-154.

MONTARRAS D., FISZMAN M., GROS F., 1982. Changes in tropomyosin during development of chick embryonic skeletal muscles in vivo and during differentiation of chick muscle cells in vitro. J. biol. Chem., 257, 545-548.

NABESHIMA Y.-I., FUJII-KURIYAMA Y., MURAMATSU M., OGATA K., 1982. Molecular cloning and nucleotide sequences of the complementary DNAs to chicken skeletal muscle myosin two alkali light chain mRNAs. Nucl. Acids Res., 10, 6099-6110.

OBINATA T., TAKANO-OHMURO H., MATSUDA R., 1980a. Changes in troponin T and myosin isoenzymes during developmental of normal and dystrophic chicken muscles. FEBS Lett., 120. 195-198.

OBINATA T., MASAKI T., TAKANO H., 1980b. Types of myosin light chains present during the development of fast skeletal muscle in chick embryo. J. Biochem., 87, 81-88.

ROSENBERG U. B., KUNZ G., FRISCHAUF A.-M., LEHRACH H., MAHR R., EPPENBERGER H. M., PERRIARD J.-C., 1982. Molecular cloning and expression during myogenesis of sequences coding for M-creatine kinase. Proc. nat. Acad. Sci. USA, 79, 6589-6592.

ROY R. K., SRETER F. A., SARKAR S., 1979. Changes in tropomyosin subunits and myosin light chains during development chicken and rabbit striated muscles. Dev. Biol., 69, 15-30.

SCHIBLER U., HAGENBUCHLE O., WELLAUER R. K., PITTER A. C., 1983. Two promotors of different strengths control the transcription of the mouse alpha-amylase gene Amy-1 ${ }^{\mathrm{a}}$ in the parotid gland and the liver. Cell, 33, 501-508. 